\title{
On the almost everywhere convergence of the ergodic averages $\dagger$
}

\author{
F. J. MARTÍN-REYES AND A. DE LA TORRE \\ Análisis Matemático, Facultad de Ciencias, Universidad de Málaga, \\ 29071-Málaga, Spain
}

(Received 18 December 1987)

\begin{abstract}
Let $(X, \mathscr{F}, \nu)$ be a finite measure space and let $T: X \rightarrow X$ be a measurable transformation. In this paper we prove that the averages $A_{n} f(x)=$ $(n+1)^{-1} \sum_{0 \leq i \leq n} f\left(T^{i} x\right)$ converge a.e. for every $f$ in $L^{p}(d \nu), 1<p<\infty$, if and only if there exists a measure $\gamma$ equivalent to $\nu$ such that the averages apply uniformly $L^{p}(d \nu)$ into weak- $L^{p}(d \gamma)$. As a corollary, we get that uniform boundedness of the averages in $L^{p}(d \nu)$ implies a.e. convergence of the averages (a result recently obtained by Assani). In order to do this, we first study measures $\nu$ equivalent to a finite invariant measure $\mu$ and we prove that $\sup _{n \geq 0} A_{n}(d \nu / d \mu)^{-1 /(p-1)}<\infty$ a.e. is a necessary and sufficient condition for the averages to converge a.e. for every $f$ in $L^{p}(d \nu)$.
\end{abstract}

\section{Introduction}

Let $(X, \mathscr{F}, \nu)$ be a finite measure space and let $T: X \rightarrow X$ be a null-preserving transformation. We consider the operator $T$, acting on measurable functions and denoted by the same letter,

$$
T f(x)=f(T x)
$$

Associated with $T$ we have the averages

$$
A_{n} f=(n+1)^{-1} \sum_{i=0}^{n} T^{i} f
$$

and the maximal operator

$$
M f=\sup _{n \geq 0} A_{n}|f| \text {. }
$$

Assani has proved [1] that if the averages are uniformly bounded in $L^{p}(d \nu)$, $1<p<\infty$, then the sequence $\left\{A_{n} f\right\}$ converges a.e. for every $f$ in $L^{p}(d \nu)$. We prove in this paper that a.e. convergence of the averages follows from a condition weaker than uniform boundedness of the averages. In fact, we have the following characterization (Theorem 3.1): The averages $A_{n} f$ converge a.e. for every $f$ in $L^{p}(d \nu), 1<p<\infty$, if and only if there exists a measure $\gamma$ equivalent to $\nu$ such that

$$
\sup _{n \geq 0} \gamma\left(\left\{x:\left|A_{n} f(x)\right|>\lambda\right\}\right) \leq \lambda^{-p} \int_{X}|f|^{p} d \nu
$$

† This research has been supported by C.A.I.C.Y.T. Grant (PB85-0434). 
for every $\lambda>0$ and any function in $L^{p}(d \nu)$. Of course, Assani's result is then a consequence of ours.

In $\S 2$, we study the problem for measures $\nu$ equivalent to an invariant measure $\mu$ and we give a characterization (Theorem 2.9), in terms of the Radon-Nikodym derivative $d \nu / d \mu$, of the measure $\nu$ for which the a.e. convergence of the averages holds for any $f$ in $L^{p}(d \nu)$ (the problem for $p=1$ was studied in [7]; see also [5] and [11] for Hopf's averages). Finally, in $\S 3$ we get the theorem for general measures $\nu$.

Throughout this paper, $C$ will be a positive constant not necessarily the same at each occurrence, and $p$ and $q$ will denote numbers bigger than 1 such that $p+q=p q$. $\mathbf{N}$ will stand for the set of natural numbers and, if $s$ and $k$ are natural numbers, $[s, k]$ will be the set $\{i \in \mathbf{N}: s \leq i \leq k\}$. Finally, $\chi_{A}$ will be the characteristic function of the set $A$ and we will consider two sets as 'equal' if they agree up to a set of measure zero.

\section{The measure-preserving case}

Let $(X, \mathscr{F}, \mu)$ be a finite measure space and let $T: X \rightarrow X$ be a measure preserving transformation. In order to state and prove the first result we will need some definitions and previous results.

Definition 2.1. Let $k$ be a natural number. The measurable subset $B$ is the base of a rectangle of length $k+1$ if $T^{-i} B \cap T^{-j} B=\varnothing, i \neq j, 0 \leq i, j \leq k$.

LEMMA 2.2. Let $k$ be a natural number. Then, there exists a countable family $\left\{B_{i}: i \in \mathbf{N}\right\}$ of sets such that

(i) $X=\bigcup_{i=0}^{\infty} B_{i}$

(ii) $B_{i} \cap B_{j}=\varnothing$ if $i \neq j$

(iii) For every $i, B_{i}$ is the base of a rectangle of length $1+s(i)$ with $0 \leq s(i) \leq k$, and such that if $s(i)<k$ then $T^{-1-s(i)} A=A$ for every measurable subset $A$ of $B_{i}$. Consequently, for every measurable subset $A$ of $B_{i}$

$$
\sum_{j=0}^{k} \chi_{T^{-i} A} \leq C(i) \sum_{j=0}^{s(i)} \chi_{T^{-i} A}=C(i) \chi \cup_{j=0}^{s(i)} T^{-j} A=2 \sum_{j=0}^{k} \chi_{T^{-i} A}
$$

where $C(i)$ is the least integer bigger than or equal to $(k+1)(1+s(i))^{-1}$.

The proof of this lemma is the same as the proof of Lemma 2.10 in [5] but changing $T^{h}$ by $T^{-h}$. Lemma 2.2 has already been used in [7].

Definition 2.4. Let $a$ be a function on N. We define the maximal function $\mathrm{M}^{+} a$ on $\mathbf{N}$ by

$$
M^{+} a(j)=\sup _{n \geq 0}(n+1)^{-1} \sum_{i=0}^{n}|a(i+j)| \text {. }
$$

THEOREM 2.5. Let $u$ and $v$ be positive functions on $\mathbf{N}$. There exists $C>0$ such that

$$
\sum_{\left\{j: M^{+} a(j)>\lambda\right\}} u(j) \leq C \lambda^{-p} \sum_{j=0}^{\infty}|a(j)|^{p} v(j)
$$


for every $\lambda>0$ and any positive function $a$ on $\mathbf{N}$ if and only if the pair of functions $(u, v)$ satisfies the following condition

$A_{p}^{+}:$There exists $D>0$ such that

$\sum_{j=0}^{s} u(i+j)\left(\sum_{j=s}^{k} v^{1-q}(i+j)\right)^{p-1} \leq D(k+1)^{p}$ for all natural numbers $i, s$ and $k$ with $s \leq k$. where $C$ and $D$ are functions of $p$ and of each other alone.

THEOREM 2.6. Let $u$ and $v$ be positive functions on $\mathbf{N}$. There exists $C>0$ such that

$$
\sum_{j=0}^{\infty}\left|M^{+} a(j)\right|^{p} u(j) \leq C \sum_{j=0}^{\infty}|a(j)|^{p} v(j)
$$

for every positive function $a$ on $\mathbf{N}$ if and only if the pair of functions $(u, v)$ satisfies the following condition

$S_{p}^{+}:$There exists $D>0$ such that

$\sum_{j=s}^{k}\left|M^{+}\left(v^{1-q} \chi_{[s, k]}\right)(j)\right|^{p} u(j) \leq D \sum_{j=s}^{k} v^{1-q}(j) \quad$ for all natural numbers $s$ and $k$ with $s \leq k$. where $C$ and $D$ are functions of $p$ and of each other alone.

For the proofs of Theorems 2.5 and 2.6 just look at the proofs in [12] or in [6] and write them in the set of the natural numbers.

LEMMA 2.7. Let $(X, \mathscr{F}, \nu)$ be a finite measure space and let $\left\{\mathscr{F}_{n}\right\}$ be a sequence of $\sigma$-algebras such that $\mathscr{F}=\mathscr{F}_{0} \supset \mathscr{F}_{1} \supset \mathscr{F}_{2} \supset \cdots$. Let $\mathscr{F}_{\infty}=\bigcap_{n} \mathscr{F}_{n}$ and denote by $E_{n}$ the conditional expectation with respect to $\mathscr{F}_{n}$. If $\left\{f_{n}\right\}$ is an a.e. convergent sequence of functions such that $\left|f_{n}\right| \leq C$ a.e. and $f$ is the a.e. limit of $f_{n}$ then $E_{\infty} f$ is the a.e. limit of $E_{n} f_{n}$.

Proof. It follows from Theorem 7.6 in [4, p. 66], and the decreasing martingale theorem (see for example [9]).

Before stating and proving our main result in this section we still need some notation.

Let $f$ be a measurable function on $X$. For every $x \in X$ we will consider the function $f_{x}$ on $\mathbf{N}$ defined by $f_{x}(i)=f\left(T^{i} x\right)$. Now, we are ready to state and prove our main result in this section.

THEOREM 2.8. Let $V$ be a positive measurable function on $X$. The following are equivalent:

(a) The sequence $\left\{A_{n} f\right\}$ converges a.e. for all $f$ in $L^{p}(V d \mu)$.

(b) There exists a positive measurable function $U$ such that

$$
\sup _{n \geqslant 0} \int_{X}\left|A_{n} f\right|^{p} U d \mu \leq \int_{X} \mid f^{p} V d \mu
$$

for all $f$ in $L^{p}(V d \mu)$. 
(c) There exists a positive measurable function $U$ such that

$$
\sup _{n \geq 0} \int_{\left\{x \in X:\left|A_{n} f(x)\right|>\lambda\right\}} U d \mu \leq \lambda^{-p} \int_{X}|f|^{p} V d \mu
$$

for all $\lambda>0$ and any $f$ in $L^{p}(V d \mu)$.

(d) $M f(x)<\infty$ a.e. for all $f$ in $L^{p}(V d \mu)$.

(e) There exists a positive measurable function $U$ such that

$$
\int_{\{x \in X: M f(x)>\lambda\}} U d \mu \leq \lambda^{-p} \int_{X}|f|^{p} V d \mu
$$

for all $\lambda>0$ and any $f$ in $L^{p}(V d \mu)$.

(f) There exists a positive measurable function $U$ such that

$$
\int_{X}|M f|^{p} U d \mu \leq \int_{X}|f|^{p} V d \mu
$$

for all $f$ in $L^{p}(V d \mu)$.

(g) $M V^{1-q}(x)<\infty$ a.e.

(h) There exists a positive measurable function $U$ such that for almost all $x$ in $X$ the pair $\left(U_{x}, V_{x}\right)$ satisfies $A_{p}^{+}$with a constant independent of $x$.

(i) There exists a positive measurable function $U$ such that for almost all $x$ in $X$ the pair $\left(U_{x}, V_{x}\right)$ satisfies $S_{p}^{+}$with a constant independent of $x$.

Proof. The following implications are clear: (a) $\Rightarrow(\mathrm{d}),(\mathrm{e}) \Rightarrow(\mathrm{c}),(\mathrm{f}) \Rightarrow(\mathrm{e}),(\mathrm{f}) \Rightarrow(\mathrm{d})$, $(f) \Rightarrow(b),(b) \Rightarrow$ (c) and (i) $\Rightarrow(h)$. Then, it will suffice to prove (d) $\Rightarrow(a),(d) \Rightarrow(e)$, $(\mathrm{c}) \Rightarrow(\mathrm{g}),(\mathrm{g}) \Rightarrow(\mathrm{i}),(\mathrm{i}) \Rightarrow(\mathrm{f})$ and $(\mathrm{h}) \Rightarrow(\mathrm{e})$.

Statement (a) follows from (d) by Banach's Principle since $L^{p}(V d \mu) \cap L^{1}(d \mu)$ is dense in $L^{p}(V d \mu)$ and the averages $A_{n} f$ converge a.e. for all $f$ in $L^{1}(d \mu)$.

On the other hand, (d) implies that $M$ is a continuous sublinear operator from $L^{p}(V d \mu)$ to $L^{0}(d \mu)$ where $L^{0}(d \mu)$ is the space of all measurable functions provided with the topology of the convergence in measure (see [3, p. 10, Theorem 1.1.1]). Then (e) follows from Nikishin's theorem [2, p. 536]. It is also easy to see that (g) implies (i) if we take $U=\left(M V^{1-q}\right)^{-p} V^{1-q}$. To prove the other implications we need to work harder.

(c) $\Rightarrow(\mathrm{g})$. We may assume $U \leq 1$. It follows from (c) that Kolmogorov's condition holds [10, p. 190], i.e. there exists a constant $C>0$ such that for any measurable set $F$ and any $f$ in $L^{p}(V d \mu)$

$$
\sup _{n \geq 0}\left(\int_{F} \mid A_{n} f^{p / 2} U d \mu\right)^{2} \leq C \int_{F} U d \mu \int_{X}|f|^{p} V d \mu \leq C \mu(F) \int_{X}|f|^{p} V d \mu .
$$

Now, fix a natural number $k$ and let $\left\{B_{i}: i \in \mathbf{N}\right\}$ be the sequence given by Lemma 2.2 for $k$ (in the proof we will use the notation in the lemma). For fixed $B_{i}$ we take any measurable set $A$ contained in $B_{i}$. Let $R_{1}=\bigcup_{0 \leq j \leq k} T^{-j} A$ and $R_{2}=\bigcup_{k \leq j \leq 2 k} T^{-j} A$ (keep in mind that $R_{1}=\bigcup_{0 \leq j \leq s(i)} T^{-j} A$ and $R_{2}=\bigcup_{0 \leq j \leq s(i)} T^{-j-k} A$ ) and consider for each natural $N$ the function $\sigma_{N}=\min (\sigma, N)$ where $\sigma=V^{i-q}$. Then if we apply 
(2.9) with $F=R_{2}, f=\sigma_{N X_{R_{1}}}$ and $n=2 k$ we have

$$
\left(\int_{R_{2}}\left|A_{2 k}\left(\sigma_{N} \chi_{R_{1}}\right)\right|^{p / 2} U d \mu\right)^{2} \leq C \mu\left(R_{2}\right) \int_{R_{1}} \sigma_{N} d \mu .
$$

On the other hand, (2.3) and the fact that $T$ preserves $\mu$ give

$$
\begin{array}{r}
\sum_{j=k}^{2 k} \int_{T^{-2 k} A}\left|A_{2 k}\left(\sigma_{N \chi R_{1}}\right)\right|^{p / 2}\left(T^{2 k-j} x\right) U\left(T^{2 k-j} x\right) d \mu \\
\leq C(i) \int_{R_{2}}\left|A_{2 k}\left(\sigma_{N X_{R_{1}}}\right)\right|^{p / 2}(x) U(x) d \mu \\
C(i) \mu\left(R_{2}\right) \leq 2(k+1) \mu\left(T^{-2 k} A\right) \\
C(i) \int_{R_{1}} \sigma_{N} d \mu \leq 2 \sum_{j=k}^{2 k} \int_{T^{-2 k} A} \sigma_{N}\left(T^{j} x\right) d \mu .
\end{array}
$$

We have also

$$
(2 k+1)^{-1} \sum_{i=k}^{2 k} \sigma_{N}\left(T^{i} x\right) \leq A_{2 k}\left(\sigma_{N \chi_{R_{1}}}\right)\left(T^{2 k-j} x\right), \quad\left(x \in T^{-2 k} A, k \leq j \leq 2 k\right) .
$$

Then, (2.14), (2.13), (2.10), (2.12) and (2.11) give

$$
\begin{aligned}
\left(\int_{T^{-2 k} A} \mid(2 k\right. & \left.+1)\left.^{-1} \sum_{i=k}^{2 k} \sigma_{N}\left(T^{i} x\right)\right|^{p / 2} \sum_{j=0}^{k} U\left(T^{j} x\right) d \mu\right)^{2} \\
\leq C(k+1) \mu\left(T^{-2 k} A\right) & \sum_{j=k}^{2 k} \int_{T^{-2 k} A} \sigma_{N}\left(T^{j} x\right) d \mu .
\end{aligned}
$$

Let $E_{n}$ be the conditional expectation with respect to $\mathscr{F}_{n}\left(\mathscr{F}_{n}=T^{-n} \mathscr{F}_{,} \mathscr{F}_{\infty}=\right.$ $\left.\bigcap_{n \geq 0} \mathscr{F}_{n}\right)$. Then the last inequality can be written in the following way

$$
\begin{aligned}
&\left(\int _ { T ^ { - 2 k } A } E _ { 2 k } \left(\left((2 k+1)^{-1}\right.\right.\right.\left.\left.\left.\sum_{i=k}^{2 k} T^{i} \sigma_{N}\right)^{p / 2} \sum_{j=0}^{k} T^{j} U\right) d \mu\right)^{2} \\
& \leq C(k+1) \mu\left(T^{-2 k} A\right) \int_{T^{-2 k} A} E_{2 k}\left(\sum_{j=k}^{2 k} T^{j} \sigma_{N}\right) d \mu .
\end{aligned}
$$

and therefore for any subset $F \in \mathscr{F}_{2 k}$ we have

$$
\left(\frac{1}{\mu(F)} \int_{F} f_{i} d \mu\right)^{2} \leq \frac{C}{\mu(F)} \int_{F} g_{i} d \mu
$$

where

$$
f_{i}=E_{2 k}\left(\left((2 k+1)^{-1} \sum_{j=k}^{2 k} T^{j} \sigma_{N}\right)^{p / 2} \sum_{j=0}^{k} T^{j} U\right) \chi_{T^{-2 k} B_{i}}
$$

and

$$
g_{i}=E_{2 k}\left((k+1) \sum_{j=k}^{2 k} T^{j} \sigma_{N}\right) \chi_{T^{-2 k} B_{i}} .
$$

Observe that $f_{i}$ and $g_{i}$ are $\mathscr{F}_{2 k}$-measurable functions. Then a standard argument proves that

$$
f_{i}^{2} \leq C g_{i} \text { a.e. }
$$


(check it for characteristic functions $f_{i}$ and $g_{i}$, then for simple functions and finally for positive functions). Summing over $i$ in the last inequality we have

$$
\begin{gathered}
\left(E_{2 k}\left(\left((2 k+1)^{-1} \sum_{j=k}^{2 k} T^{j} \sigma_{N}\right)^{p / 2}(k+1)^{-1} \sum_{j=0}^{k} T^{j} U\right)\right)^{2} \\
\leq C E_{2 k}\left((k+1)^{-1} \sum_{j=k}^{2 k} T^{j} \sigma_{N}\right)
\end{gathered}
$$

Since $\sigma_{N}$ and $U$ are functions in $L^{1}(d \mu)$ the averages $A_{n} \sigma_{N}$ and $A_{n} U$ converge a.e. to invariant functions $E \sigma_{N}$ and $E U$ respectively. Then Lemma 2.7 together with 2.16 give $\left(E \sigma_{N}\right)^{p-1}(E U)^{2} \leq C$ a.e. Since $C$ is independent of $N$ and $E U \neq 0$ a.e. we get $\lim _{N \rightarrow \infty} E \sigma_{N}<\infty$ a.e. Therefore, up to a set of measure zero, we have

$$
X=\bigcup_{s=1}^{\infty} X_{s}, \quad X_{s}=\left\{x: \lim _{N \rightarrow \infty} E \sigma_{N} \leq s\right\}
$$

Of course, each $X_{s}$ is invariant and thus

$$
\int_{X_{s}} \sigma d \mu=\lim _{N \rightarrow \infty} \int_{X_{s}} E \sigma_{N} d \mu=\int_{X_{s}} \lim _{N \rightarrow \infty} E \sigma_{N} d \mu \leq s \mu\left(X_{s}\right)<\infty
$$

Hence $\sigma \in L^{1}\left(X_{s}, d \mu\right)$ and since $X_{s}$ is invariant we have $M \sigma(x)<\infty$ a.e. in $X_{s}$. This proves the implication.

(i) $\Rightarrow$ (f). We consider the truncated maximal operator $M_{L}(L \geq 1), M_{L} f=$ $\sup _{n \leq L} A_{n}|f|$. Since $T$ preserves the measure $\mu$ we have

$$
\int_{X}\left|M_{L} f\right|^{p} U d \mu=(k+1)^{-1} \sum_{i=0}^{k} \int_{X}\left|M_{L} f\left(T^{i} x\right)\right|^{p} U\left(T^{i} x\right) d \mu .
$$

If $H^{x}=f_{x} \chi_{[0, L+k]}$ then the right-hand side in (2.17) is smaller than or equal to

$$
\int_{X}(k+1)^{-1} \sum_{i=-\infty}^{\infty}\left|M^{+} H^{x}(i)\right|^{p} V_{x}(i) d \mu \text {. }
$$

By (2.6), the integrand is dominated by

$$
C(k+1)^{-1} \sum_{i=0}^{k+L}\left|f\left(T^{i} x\right)\right|^{p} U\left(T^{i} x\right)
$$

If we put these inequalities in (2.17) we get

$$
\int_{X}\left|M_{L} f\right|^{p} U d \mu \leq C(k+L+1)(k+1)^{-1} \int_{X}|f|^{p} V d \mu .
$$

If we let $k$ and then $L$ tend to infinity we obtain (f).

Finally, (e) follows from (h) by transference arguments in the same way that (f) follows from (i) but using Theorem 2.5 instead of Theorem 2.6.

Remarks: 2.18. An easy way to get $M V^{1-q}<\infty$ a.e. is to take $V^{1-q}$ in $L^{1}(d \mu)$, actually it is the only way for ergodic $T$. But this means that $L^{p}(V d \mu) \subset L^{1}(d \mu)$ and thus the convergence follows from the individual ergodic theorem. We will now give an example which shows that it is possible to get $M V^{1-q}<\infty$ a.e. and $V^{1-q} \notin L^{1}(d \mu)$. 
Let $X=\{(n, i) \in \mathbf{Z} \times \mathbf{Z}: 1 \leq n, 1 \leq i \leq n\}$ and let $T$ be the transformation given by $T(n, i)=(n, i+1)$ if $1 \leq i \leq n$ and $T(n, n)=(n, 1)$. Consider the measure $\mu$ determined by $\mu\{(n, i)\}=2^{-n}$. Finally, let $\nu=V d \mu$ where $V(n, i)=2^{-i}$. It is clear that $\mu$ is a finite invariant measure, $\nu$ is a finite measure equivalent to $\mu, M V^{1-q}<\infty$ and $V^{1-q}$ is not in $L^{1}(d \mu)$.

2.19. The proofs of the implications $(\mathrm{a}) \Rightarrow(\mathrm{d}) \Rightarrow(\mathrm{e}) \Rightarrow(\mathrm{c}) \Rightarrow(\mathrm{g})$ do not use Theorems 2.5 and 2.6. We will now give a proof of $(\mathrm{g}) \Rightarrow$ (a) also without using 2.5 and 2.6. In this way we prove the equivalence of (a), (c), (d), (e) and (g) without using conditions $A_{p}^{+}$and $S_{p}^{+}$. Therefore, the importance of 2.6 in Theorem 2.8 is that it allows to get a strong type inequality (and thus dominated convergence) and not only a weak type inequality as Nikishin's Theorem ensures.

$A$ direct proof of $(\mathrm{g}) \Rightarrow(\mathrm{a})$. By (g) we have $X=\bigcup_{k \geq 1} X_{k}$ where $X_{k}=$ $\left\{x: \limsup _{n \rightarrow \infty} A_{n} V^{1-q}(x)<k\right\}$. Of course, $X_{k}$ is invariant. On the other hand, using Holder's inequality, a truncation argument and Birkhoff's ergodic theorem we have

$$
\begin{aligned}
\int_{X_{k}}|f| d \mu & \leq\left(\int_{X_{k}}|f|^{p} V d \mu\right)^{1 / p}\left(\int_{X_{k}} V^{1-q} d \mu\right)^{1 / q} \\
& \leq\left(\int_{X_{k}}|f|^{p} V d \mu\right)^{1 / p}\left(k \mu\left(X_{k}\right)\right)^{1 / q} .
\end{aligned}
$$

Therefore $L^{p}\left(X_{k}, V d \mu\right) \subset L^{1}\left(X_{k}, V d \mu\right)$. Then (a) follows from this inclusion and the individual ergodic theorem.

\section{The a.e. convergence in the general case}

This section is devoted to establishing a characterization of the finite measures $\nu$ for which the individual ergodic theorem holds in $L^{p}(d \nu), 1<p<\infty$.

TheOREM 3.1. Let $(X, \mathscr{F}, \nu)$ be a finite measure space and let $T: X \rightarrow X$ be a nullpreserving transformation. The following are equivalent:

(a) There exists a (finite) measure $\gamma$ equivalent to $\nu$ such that

$$
\sup _{n \geq 0} \gamma\left(\left\{x:\left|A_{n} f(x)\right|>\lambda\right\}\right) \leq \lambda^{-p} \int_{X}|f|^{p} d \nu
$$

for all $\lambda>0$ and any $f$ in $L^{p}(d \nu)$.

(b) There exists a measure $\gamma$ equivalent to $\nu$ such that

$$
\gamma(\{x: M f(x)>\lambda\}) \leq \lambda^{-p} \int_{X}|f|^{p} d \nu
$$

for all $\lambda>0$ and any $f$ in $L^{p}(d \nu)$.

(c) The sequence $\left\{A_{n} f\right\}$ converges a.e. for all $f$ in $L^{p}(d \nu)$.

(d) $M f(x)<\infty$ a.e. for all $f$ in $L^{p}(d \nu)$.

Proof. The implications (b) $\Rightarrow(\mathrm{a})$ and $(\mathrm{c}) \Rightarrow(\mathrm{d})$ are clear and (b) follows from (d) by Nikishin's theorem (see the proof of $(d) \Rightarrow(e)$ in Theorem 2.8). Hence we have only to prove $(\mathrm{a}) \Rightarrow(\mathrm{c})$. 
(a) $\Rightarrow(\mathrm{c})$. Since (a) holds and the averages $A_{n}$ are uniformly bounded from $L^{\infty}(d \nu)$ to $L^{\infty}(d \gamma)$ we have by Marcinkiewicz's interpolation theorem

$$
\sup _{n \geq 0} \int_{X}\left|A_{n} f\right|^{2 p} d \gamma \leq C \int_{X}|f|^{2 p} d \nu
$$

for every $f$ in $L^{2 p}(d \nu)$. Let $L$ be a Banach's limit, i.e. a functional on the set of bounded sequences such that if $\left\{s_{k}\right\}$ and $\left\{t_{k}\right\}$ are bounded sequences

$$
\begin{gathered}
\underset{k \rightarrow \infty}{\liminf } s_{k} \leq L\left(\left\{s_{k}\right\}\right) \leq \limsup _{k \rightarrow \infty} s_{k} \\
\text { if } s_{k}=t_{k+1} \text { then } L\left(\left\{s_{k}\right\}\right)=L\left(\left\{t_{k}\right\}\right) .
\end{gathered}
$$

For every measurable set $E$ we consider the sequence $\left\{\int_{X} A_{n} \chi \chi_{E} d \gamma\right\}$ which is a bounded sequence by (3.2). We then define

$$
\mu(E)=L\left(\left\{\int_{X} A_{n} \chi_{E} d \gamma\right\}\right) .
$$

It is clear that $\mu$ is a finitely additive measure. On the other hand, by (3.3) and (3.2) we have

$$
\mu(E) \leq C(\gamma(X))^{1 / q^{\prime}}(\nu(E))^{1 / 2 p}<\infty \quad \text { where } q^{\prime}+2 p=2 p q^{\prime} .
$$

Hence $\mu(X)<\infty$ and it follows from (3.5) and the fact that $\nu$ is a finite measure that $\lim _{k \rightarrow \infty} \mu\left(E_{k}\right)=0$ for every sequence of measurable sets $\left\{E_{k}\right\}$ with $E_{k} \supset E_{k+1}$ and $\bigcap_{k} E_{k}=\varnothing$. Thus $\mu$ is a (countably additive) measure. It is easy to see that $\mu$ is invariant and absolutely continuous with respect to $\nu$. Let $v$ be the Radon-Nikodym derivative $d \mu / d \nu$ and let $D$ be the set $\{x: v(x) \neq 0\}$. Observe that $T^{-1} D=X$ a.e. ( $\mu$ ) since $\mu$ is invariant and $\mu(X-D)=0$. Consider the set $Y=\bigcap_{n \geq 0} T^{-n} D$. It is clear that $\mu(X-Y)=0$ and the restriction of $T$ to $Y,\left.T\right|_{Y}$, applies $Y$ in $Y$. Furthermore, $\left.\mu\right|_{Y}$ is invariant with respect to $\left.T\right|_{Y}$. Therefore we have that $\left.\nu\right|_{Y}$ is equivalent to the invariant measure $\left.\mu\right|_{Y}$. From (a) in this theorem and the equivalence of (c) and (a) in Theorem 2.8 it follows that $A_{n}\left(f_{\chi_{Y}}\right)(x)$ converges a.e. in $Y$ for every $f \in L^{p}(d \nu)$. Since $A_{n}\left(f \chi_{X-Y}\right)(x)=0$ for every $x$ in $Y$ we have that for every $f$ in $L^{p}(d \nu)$ the averages $A_{n} f(x)$ converge a.e. in $Y$. To prove the convergence in $X-Y$ it suffices to establish the following property (the idea of this part of the proof is in [1]):

3.6. For $\nu$-almost all $x$ in $X$ there exists $n$ such that $T^{n} x \in Y$.

We will now prove 3.6. If 3.6 does not hold then there exists $B$ with $\nu(B)>0$ such that for every $i, B \subset T^{-i}(X-Y)$. Then for every $n$

$$
\gamma(B) \leq(n+1)^{-1} \sum_{i=0}^{n} \gamma\left(T^{-i}(X-Y)\right)
$$

and therefore

$$
\gamma(B) \leq \mu(X-Y)=0
$$

and this finishes the proof because $\gamma$ is equivalent to $\nu$. 
Once Theorem 3.1 has been proved, the result of Assani mentioned in the Introduction follows as a corollary.

Corollary 3.6. (See [1].) Let $(X, \mathscr{F}, \mu)$ be a finite measure space and let $T: X \rightarrow X$ be a null-preserving transformation. If $\sup _{n \geq 0}\left\|A_{n}\right\|_{p}<\infty$ then for every $f$ in $L_{p}(d \nu)$ the sequence of the averages $A_{n} f$ converges a.e.

Remark 3.7. Observe that uniform boundedness of the averages is certainly a stronger condition than the conditions in Theorem 3.1. In order to see this, we consider the example in Remark 2.18. We proved in that example that $M V^{1-q}<\infty$ and therefore, by Theorem 2.8 , the averages converge a.e. However, $V$ does not satisfy $A_{p}^{+}$and thus (see [8]) the averages are not uniformly bounded in $L^{p}(d \nu)$.

\section{REFERENCES}

[1] I. Assani. Quelques resultats sur les opérateurs positifs à moyennes bornées dan $L_{p}$. Ann. Scien. Clermont-Ferrand 85, Probabilités, Fasc. 3 (1985), 65-72.

[2] J. García-Cuerva \& J. L. Rubio de Francia. Weighted Norm Inequalities and Related Topics. North Holland: Amsterdam, 1985.

[3] M. de Guzmán. Real Variable Methods in Fourier Analysis. North-Holland: Amsterdam, 1981.

[4] U. Krengel. Ergodic Theorems. Walter de Gruyter: 1985.

[5] F. J. Martín-Reyes. Inequalities for the maximal function and convergence of the averages in weighted $L_{p}$-spaces. Trans. Amer. Math. Soc. 296 (1986), 61-82.

[6] F. J. Martín-Reyes, P. Ortega Salvador \& A. de la Torre. Weighted inequalities for one-sided maximal functions. To appear in T.A.M.S.

[7] F. J. Martín-Reyes \& A. de la Torre. Weighted weak type inequalities for the ergodic maximal function and the pointwise ergodic theorem. Studia Math. 87 (1987), 33-46.

[8] F. J. Martín-Reyes \& A. de la Torre. The dominated ergodic estimate for mean bounded, invertible, positive operators. Proc. Amer. Math. Soc. 104 (1988) 69-75.

[9] W. Parry. Topics in Ergodic Theory. Cambridge University Press: Cambridge, 1981.

[10] C. Sadosky. Interpolation of Operators and Singular Integrals. Marcel Dekker: New York, 1979.

[11] R. Sato. On a ratio ergodic theorem. Studia Math. 87 (1987), 47-52.

[12] E. Sawyer. Weighted inequalities for the one-sided Hardy Littlewood maximal functions. Trans. Amer. Math. Soc. 297 (1986), 53-61. 